\title{
Application of the Boundary Element Method for the Simulation of Two-dimensional Viscous Incompressible Flow
}

\author{
Dzmitry Prybytak \\ Gdańsk University of Technology, Faculty of Civil and Environmental Engineering, ul. G. Narutowicza \\ 11/12, 80-233 Gdańsk, Poland, e-mail: dzmpryby@student.pg.gda.pl
}

(Received October 14, 2014; revised December 16, 2014)

\begin{abstract}
The paper presents the application of an indirect variant of the boundary element method (BEM) to solve the two-dimensional steady flow of a Stokes liquid. In the BEM, a system of differential equations is transformed into integral equations. This makes it possible to limit discretization to the border of the solution. Numerical discretization of the computational domain was performed with linear boundary elements, for which a constant value of unknown functions was assumed. The verification was carried out for the case of flow in a square cavity with one moving wall. The results obtained show that the use of approximations by simple linear functions is relatively easy for different shapes of the area, but the result may be affected by significant errors.
\end{abstract}

Key words: mathematical modeling, numerical solution, boundary element method, flow of a Stokes liquid

\section{Introduction}

Mathematical modeling of liquid flow is a part of computational fluid dynamics. Mathematical equations representing the fundamental laws of physics (e.g. the Navier-Stokes (NS) equations) have been well known for years. However, their solution is practically impossible for the scale of any real-life case. So, for numerical simulation,simplified models are usually solved. Simplifications can apply both to the properties of liquids and to some of the impacts on their body.In many cases, we can consider a Newtonian fluid with a constant density and viscosity, and the forces of inertia can be neglected during its motion.

The equations describing the motion of the liquid are a mathematical expression of the laws of conservation of mass and momentum, supplemented by the constitutive equation defining the relationship between the tangential stress and viscosity, known as Newton's hypothesis. In the general case we can write them in the following vector form: 


$$
\begin{gathered}
\rho \cdot \frac{\partial \vec{u}}{\partial t}+\rho \cdot \vec{u} \cdot(\nabla \vec{u})=-\nabla p+\mu \cdot \Delta \vec{u}, \\
\nabla \cdot \vec{u}=0,
\end{gathered}
$$

where:

$\rho \quad-\quad$ density of the fluid,

$t \quad-$ time variable,

$\vec{u} \quad-$ velocity vector, $\vec{u}=\left[u_{x}, u_{y}, u_{z}\right]$,

$p$ - pressure,

$\mu \quad-$ dynamic viscosity coefficient $(\mu=$ const $)$,

$\nabla \quad-$ differential operator nabla,

$$
\nabla=\frac{\partial}{\partial x} \boldsymbol{i}+\frac{\partial}{\partial y} \boldsymbol{j}+\frac{\partial}{\partial z} \boldsymbol{k},
$$

$\Delta \quad-\quad$ Laplace differential operator,

$$
\Delta=\frac{\partial^{2}}{\partial x^{2}}+\frac{\partial^{2}}{\partial y^{2}}+\frac{\partial^{2}}{\partial z^{2}}
$$

$\boldsymbol{i}, \boldsymbol{j}, \boldsymbol{k}-$ unit vectors of the coordinate system of spatial axes $x, y, z$.

In order to convert the equations to dimensionless forms, we define the characteristic velocity $U_{c}$ and the characteristic length $L_{c}$ (Marin 2012). Then we can define dimensionless variables $(*-$ indicates a dimensionless variable):

$$
x^{*}=\frac{x}{L_{c}}, \quad u^{*}=\frac{u}{U_{c}}, \quad t^{*}=t \frac{U_{c}}{L_{c}}, \quad p^{*}=\frac{p L_{c}}{\mu U_{c}} .
$$

Taking into account equation (5) and multiplying both sides of equation (1) by $L_{c}^{2} / U_{c} \mu$, we obtain:

$$
\begin{gathered}
\frac{\rho U_{c} L_{c}^{2}}{\mu L_{c}} \frac{\partial \vec{u}^{*}}{\partial t^{*}}+\frac{\rho U_{c} L_{c}}{\mu} \vec{u}^{*} \cdot\left(\nabla \vec{u}^{*}\right)=-\nabla p^{*}+\Delta \vec{u}^{*}, \\
\nabla \cdot \vec{u}^{*}=0 .
\end{gathered}
$$

Then, assuming the time scale $T_{c}=L_{c} / U_{c}$ and using formula $v=\mu / \rho$, we can define dimensionless numbers:

$$
\beta=\frac{L_{c}^{2}}{v T_{c}}, \quad R e=\frac{U_{c} L_{c}}{v},
$$

where:

$$
\begin{aligned}
& R e-\text { Reynolds number, } \\
& \beta-\text { Stokes number. }
\end{aligned}
$$


Taking into account Eq. (8), we obtain the dimensionless form of the equation of conservation of momentum:

$$
\beta \frac{\partial \vec{u}^{*}}{\partial t^{*}}+\operatorname{Re} \vec{u}^{*} \cdot\left(\nabla \vec{u}^{*}\right)=-\nabla p^{*}+\Delta \vec{u}^{*} .
$$

If $R e \ll 1$ and $\beta \ll 1$, then we can skip the terms on the left side, and the NS equation (9) simplifies to the so-called linear Stokes equation, which, after conversion to the dimensional equations, can be written as follows:

$$
\begin{gathered}
\nabla p-\mu \Delta \vec{u}=0, \\
\nabla \cdot \vec{u}=0 .
\end{gathered}
$$

The resulting vector of equations (10) and (11) may be used for description of twoand three-dimensional problems. In this paper,only a two-dimensional case will be discussed.

\section{The Basic Equation of BEM}

In general, the system of equations (10) and (11) has no analytical solution, and these equations are solved by numerical methods. For purposes of this paper, the solution was obtained by the BEM. This method requires the transformation of differential equations into their integral forms. The main advantage of the BEM is the fact that only the border of the domain is subject to discretization. In the case of a two-dimensional domain, discretization applies to a one-dimensional boundary area.

The starting point for the derivation of boundary integral equations is the Lorentz principle of reciprocal identity (Pozrikidis 1992). According to this principle, for two arbitrary no-singular flows $u$ and $u^{\prime}$ with the associated stress tensors $\sigma$ and $\sigma^{\prime}$ :

$$
\frac{\partial}{\partial x_{k}}\left(u_{i}^{\prime} \sigma_{i k}-u_{i} \sigma_{i k}^{\prime}\right)=0 .
$$

Defining $u^{\prime}$ as a flow induced by a point force with intensity $g$ located at the point $x_{0}$, we can write:

$$
\begin{gathered}
u_{i}^{\prime}=\frac{1}{4 \pi \mu} G_{i j}\left(x, x_{0}\right) g_{j}, \\
\sigma_{i k}^{\prime}(x)=\frac{1}{4 \pi} T_{i j k}\left(x, x_{0}\right) g_{j},
\end{gathered}
$$

where:

$$
\begin{aligned}
& G_{i j}-\text { two-dimensional Stokeslet }(G-\text { tensor }), \\
& T_{i j k}-\text { tensor of stress fields, associated with } G
\end{aligned}
$$


their exact form will be described below.

Substituting expressions (13) and (14) into the above formula (12) and rejecting the constant $g$, we obtain:

$$
\frac{\partial}{\partial x_{k}}\left(G_{i j}\left(x, x_{0}\right) \cdot \sigma_{i k}-\mu u_{i} \cdot T_{i j k}\left(x, x_{0}\right)\right)=0 .
$$

Applying the Gauss-Ostrogradsky theorem and introducing a surface force

$$
\vec{\phi}=\sigma \cdot \vec{n},
$$

where: $\vec{n}$ - vector normal to the boundary of domain fluid flow, we obtain the integral equation:

$$
\oint_{\Gamma} \phi_{i}(x) \cdot G_{i j}\left(x, x_{0}\right) d \Gamma-\oint_{\Gamma} \mu u_{i} \cdot T_{i j k}\left(x, x_{0}\right) \cdot n_{k}(x) d \Gamma=0,
$$

where: $\Gamma$ - boundary of domain fluid flow.

Taking into account the singularity $T_{i j k}\left(x, x_{0}\right) \cdot n_{k}(x)$ (Pozrikidis 1992), for the interior domain of integration, we obtain:

$$
u_{j}\left(x_{0}\right)=-\frac{1}{4 \pi \mu} \oint_{\Gamma} \phi_{i}(x) \cdot G_{i j}\left(x, x_{0}\right) d \Gamma+\frac{1}{4 \pi} \oint_{\Gamma} u_{i} \cdot T_{i j k}\left(x, x_{0}\right) \cdot n_{k}(x) d \Gamma .
$$

For the case when $x_{0} \in \Gamma$

$$
u_{j}\left(x_{0}\right)=-\frac{1}{2 \pi \mu} \oint_{\Gamma} \phi_{i}(x) \cdot G_{i j}\left(x, x_{0}\right) d \Gamma+\frac{1}{2 \pi} \oint_{\Gamma} u_{i} \cdot T_{i j k}\left(x, x_{0}\right) \cdot n_{k}(x) d \Gamma .
$$

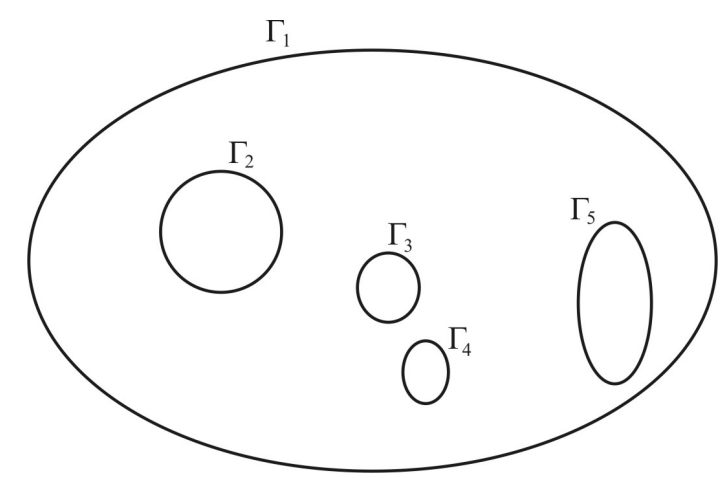

Fig. 1. Example of multi-contiguous domains $\Gamma=\bigcup_{n=1}^{5} \Gamma_{n}$ 
Because the desired velocity is expressed by a contour integral, the expressions obtained can be easily used for multi-contiguous domains (Fig. 1):

$$
\begin{gathered}
u_{j}\left(x_{0}\right)= \\
=-\frac{1}{4 \pi \mu} \sum_{n=1}^{N}\left[\oint_{\Gamma_{n}} \phi_{i}(x) \cdot G_{i j}\left(x, x_{0}\right) d \Gamma_{n}-\mu \oint_{\Gamma_{n}} u_{i} \cdot T_{i j k}\left(x, x_{0}\right) \cdot n_{k}(x) d \Gamma_{n}\right],
\end{gathered}
$$

and for the case when $x_{0} \in \Gamma$ :

$$
\begin{gathered}
u_{j}\left(x_{0}\right)= \\
=-\frac{1}{2 \pi \mu} \sum_{n=1}^{N}\left[\oint_{\Gamma_{n}} \phi_{i}(x) \cdot G_{i j}\left(x, x_{0}\right) d \Gamma_{n}-\mu \oint_{\Gamma_{n}} u_{i} \cdot T_{i j k}\left(x, x_{0}\right) \cdot n_{k}(x) d \Gamma_{n}\right] .
\end{gathered}
$$

Next, we can find Green's functions for the velocity, pressure, and stress tensor (Pozrikidis 1992), which represent a solution of the continuity equation (2) and singular Stokes equation at the point $x_{0}$ :

$$
-\nabla p+\mu \Delta \vec{u}+g \delta\left(x-x_{0}\right)=0,
$$

where: $\delta\left(x-x_{0}\right)$ - Dirac delta function.

If we move the delta function to the right side of equation (22) and define it as:

$$
\delta(\hat{x})=\frac{1}{2 \pi} \nabla^{2} \ln r
$$

where: $r=|\hat{x}|, \hat{x}=x-x_{0}$,

remembering that the pressure is a harmonic function and balancing the pressure gradient and the dimension of the delta function, we obtain:

$$
P=\frac{1}{2 \pi}(\nabla \ln r) g .
$$

Substituting (24) and (23) into (22), we obtain:

$$
\mu \nabla^{2} u=\frac{1}{2 \pi} g\left(\nabla \nabla-I \nabla^{2}\right)(\ln r),
$$

where: $I$ - identity matrix.

The expression for the velocity in terms of the scalar function $H$ will be as follows:

$$
u=\frac{1}{\mu} g\left(\nabla \nabla-I \nabla^{2}\right) H .
$$

It should be noted that the continuity equation (2) is satisfied for any $H$. Substituting (26) into (25), we obtain:

$$
\left(\nabla \nabla-I \nabla^{2}\right)\left(\nabla^{2} H-\frac{\ln r}{2 \pi}\right)=0 .
$$


Equation (27) is satisfied for any solution of the Poisson equation:

$$
\nabla^{2} H=\frac{\ln r}{2 \pi} .
$$

Taking into account the expression for the delta function (23), we obtain that $H$ is Green's function for the biharmonic equation:

$$
\nabla^{4} H=\delta\left(x-x_{0}\right)
$$

Finally, we obtain:

$$
\begin{gathered}
H=\frac{1}{8 \pi} r^{2}(\ln r-1), \\
G_{i j}=-\delta_{i j} \ln r+\frac{\hat{x}_{i} \hat{x}_{j}}{r^{2}}, \\
P_{i}=2 \frac{\hat{x}_{i}}{r^{2}},
\end{gathered}
$$

where: $P$ - pressure, associated with $G$.

Associated with (31) and (32), the stress tensor takes the following form:

$$
T_{i j k}\left(x, x_{0}\right)=-\delta_{i k} P_{j}\left(x, x_{0}\right)+\frac{\partial G_{i j}}{\partial x_{k}}\left(x, x_{0}\right)+\frac{\partial G_{i j}}{\partial x_{i}}\left(x, x_{0}\right) .
$$

Substituting $G_{i j}(31)$ and $p_{i}(32)$, we obtain:

$$
T_{i j k}\left(x, x_{0}\right)=-4 \frac{\hat{x}_{i} \hat{x}_{j} \hat{x}_{k}}{r^{2}}
$$

\section{Numerical Approximation of BEM Eequations}

Assume, that the discretization of a border area consisting of $N$ segments, we obtain boundary elements (in the 2D approach, they are sections). Along each section, the normal and tangential liquid velocity $\vec{u}=\left(u_{1}, u_{2}\right)$ or surface forces $\vec{\phi}=\left(\phi_{1}, \phi_{2}\right)$ must be given. At the same time, every point of the boundary must have two scalar values in the following variants: $\left(u_{1}, u_{2}\right),\left(\phi_{1}, \phi_{2}\right),\left(u_{1}, \phi_{1}\right),\left(u_{1}, \phi_{2}\right),\left(u_{2}, \phi_{1}\right),\left(u_{2}, \phi_{2}\right)$. In this paper, a case will be considered where only the velocity vector $\vec{u}=\left(u_{1}, u_{2}\right)$ on the border of the solutions is given, and surface forces are unknown. It is also very important that the shape of the function $\phi_{j}(x)$ is known for each boundary element and is assumed to have a constant value $\phi_{j}(x)=$ const (the simplest case).

Finally, after discretization, we obtain a new form of integral equations (19):

$$
\begin{gathered}
u_{j}\left(x_{0}^{p}\right)= \\
=-\frac{1}{2 \pi \mu} \sum_{q=1}^{N}\left[\vec{\phi}\left(\xi_{0}^{q}\right) \int_{-d a_{q}}^{d a_{q}} G_{i j}\left(x, \xi\left(\xi_{x}, 0\right)\right) d \xi_{x} \mid \frac{\vec{u}_{j}\left(x_{0}^{p}\right)}{\left|\vec{u}_{j}\left(x_{0}^{p}\right)\right|}\right]+\frac{1}{2 \pi} I_{j}\left(x_{0}^{p}\right),
\end{gathered}
$$




$$
I_{j}\left(x_{0}^{p}\right)=\sum_{q=1}^{N}\left[\left.\vec{u}\left(\xi_{0}^{q}\right) \int_{-d a_{q}}^{d a_{q}} T_{i j k}\left(x, \xi\left(\xi_{x}, 0\right)\right) \cdot(0,1) d \xi_{x}\right|_{x=x_{0}^{p}} \cdot \frac{\vec{u}_{j}\left(x_{0}^{p}\right)}{\left|\vec{u}_{j}\left(x_{0}^{p}\right)\right|}\right]
$$

where:

$$
i=1,2, j=1,2, p=1,2, \ldots, N .
$$

In equation (35) the integrals of the vectors $G_{1 j}$ and $G_{2 j}$ are equal:

$$
\begin{aligned}
& \vec{F}_{\tau}\left(x, d a_{q}\right)=\int_{-d a_{q}}^{d a_{q}} G_{1 j}\left(x, \xi\left(\xi_{x}, 0\right)\right) d \xi_{x}, \\
& \vec{F}_{n}\left(x, d a_{q}\right)=\int_{-d a_{q}}^{d a_{q}} G_{2 j}\left(x, \xi\left(\xi_{x}, 0\right)\right) d \xi_{x},
\end{aligned}
$$

and for the stress tensor:

$$
T_{i j}^{*}\left(x, d a_{q}\right)=\int_{-d a_{q}}^{d a_{q}} T_{i j k}\left(x, \xi\left(\xi_{x}, 0\right)\right) \cdot(0,1) d \xi_{x} .
$$

The solution of these integrals can be obtained analytically, and we can write:

$$
\begin{gathered}
u_{\tau}\left(x_{0}^{p}\right)=-\frac{1}{2 \pi \mu} \sum_{q=1}^{N}\left[\left[\phi_{1}\left(\xi_{0}^{q}\right) \cdot \vec{F}_{\tau}\left(x_{0}^{p}, d a_{q}\right)+\right.\right. \\
\left.\left.+\phi_{2}\left(\xi_{0}^{q}\right) \cdot \vec{F}_{n}\left(x_{0}^{p}, d a_{q}\right)\right] \cdot \vec{\tau}_{p}\right]+\frac{1}{2 \pi} I_{j}^{\Gamma}\left(x_{0}^{p}\right)_{\tau} \\
u_{n}\left(x_{0}^{p}\right)=-\frac{1}{2 \pi \mu} \sum_{q=1}^{N}\left[\left[\phi_{1}\left(\xi_{0}^{q}\right) \cdot \vec{F}_{\tau}\left(x_{0}^{p}, d a_{q}\right)+\right.\right. \\
\left.\left.+\phi_{2}\left(\xi_{0}^{q}\right) \cdot \vec{F}_{n}\left(x_{0}^{p}, d a_{q}\right)\right] \cdot \vec{n}_{p}\right]+\frac{1}{2 \pi} I_{j}^{\Gamma}\left(x_{0}^{p}\right)_{n} \\
I_{j}\left(x_{0}^{p}\right)_{\tau}=\sum_{q=1}^{N}\left[\vec{u}\left(\xi_{0}^{q}\right) \cdot T_{i j}^{*}\left(x_{0}^{p}, d a_{q}\right) \cdot \vec{\tau}_{p}\right] \\
I_{j}\left(x_{0}^{p}\right)_{n}=\sum_{q=1}^{N}\left[\vec{u}\left(\xi_{0}^{q}\right) \cdot T_{i j}^{*}\left(x_{0}^{p}, d a_{q}\right) \cdot \vec{n}_{p}\right]
\end{gathered}
$$

where: $x_{0}^{p}, \xi_{0}^{p}-$ midpoints of elements with indices $p, q$. 
Taking into account that $p=1,2, \ldots, N$, we obtain $2 N$ linear equations relative to unknown $\phi_{j}^{k}, j=1,2, k=1,2, \ldots, N$. The matrix of the resulting system of linear equations is absolutely full, and its solution has been carried out with the library cuda, which implements the parallel algorithm LU-factorization for solving a system of linear equations.

After solving the system of equations (40) and (41), when the force vector $\vec{\phi}$ is already known for each boundary element, we can calculate the velocity inside the area at any point $x_{0}$, using:

$$
\begin{gathered}
\vec{u}\left(x_{0}\right)=-\frac{1}{4 \pi \mu} \sum_{q=1}^{N}\left[\phi_{1}\left(\xi_{0}^{q}\right) \cdot \vec{F}_{\tau}\left(x_{0}, d a_{q}\right)+\phi_{2}\left(\xi_{0}^{q}\right) \cdot \vec{F}_{n}\left(x_{0}, d a_{q}\right)\right]+\frac{1}{4 \pi} I_{j}\left(x_{0}\right), \\
I\left(x_{0}\right)=\sum_{q=1}^{N} \vec{u}\left(\xi_{0}^{q}\right) \cdot T_{i j}^{*}\left(x_{0}, d a_{q}\right) .
\end{gathered}
$$

\section{Numerical Calculations and Discussion of Results}

In order to verify the adopted computational models, the case of flow in a square cavity with one moving wall was considered (Teleszewski and Sorko 2011). This case has been widely presented in the literature devoted to discrete methods of determining the Stokes flow and is a generally accepted formulation of the test tasks for these methods.

Fig. 2 presents an example of domain discretization with twelve boundary elements. Fig. 3 shows the distribution of streamlines obtained with a) 48, b) 280, c) 800 , and d) 4000 boundary elements.

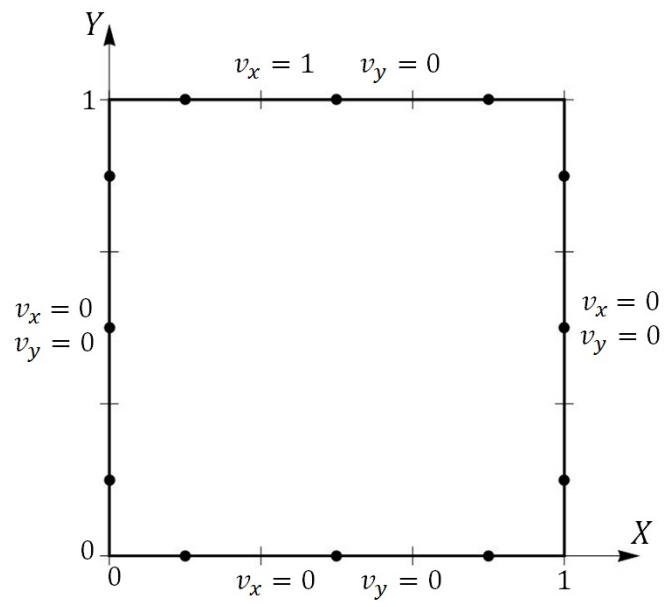

Fig. 2. Example of domain discretization with 12 boundary elements and boundary conditions relative to the velocity 
a)

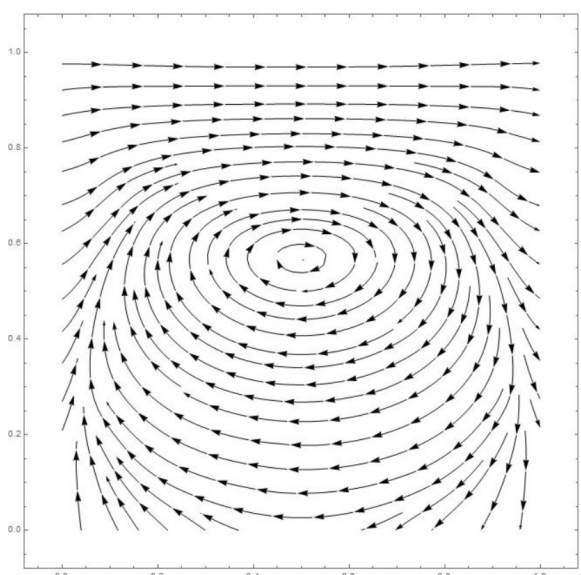

c)

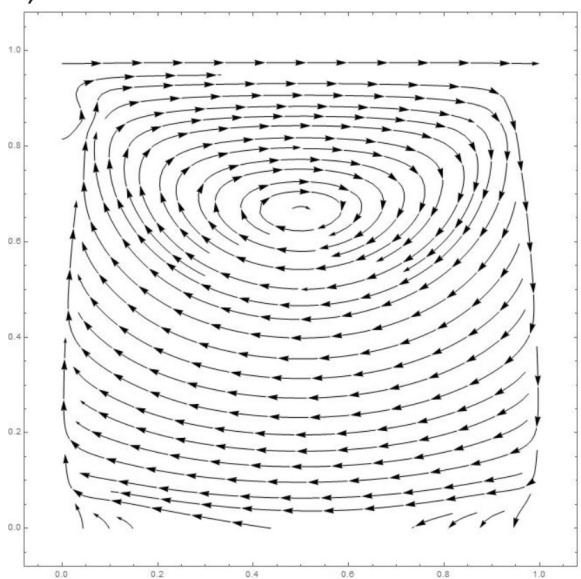

b)

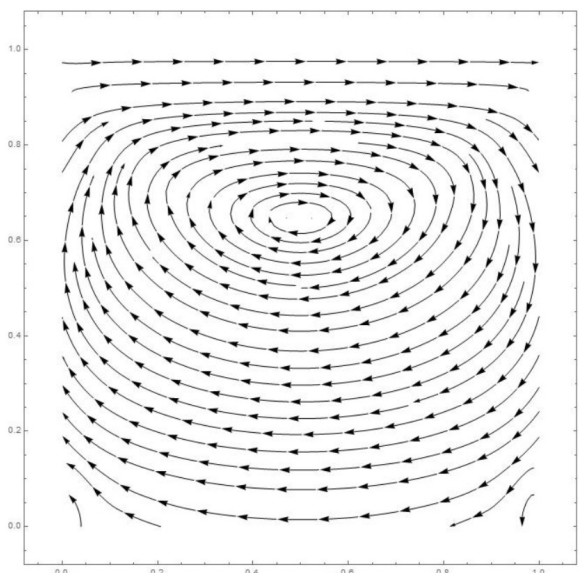

d)

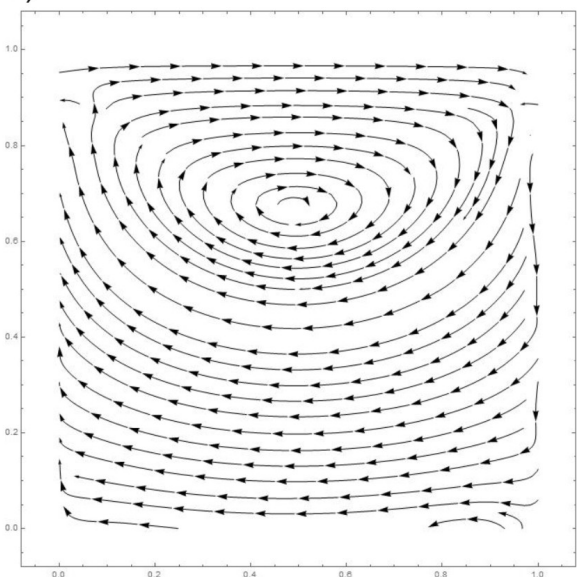

Fig. 3. Distribution of streamlines obtained with a) 48 , b) 280 , c) 800 , and d) 4000 boundary elements

Analyzing qualitatively the results presented in Fig. 3, we can see differences in the solutions obtained. They are due to the following two conditions. Firstly, they arise from the fact that boundary conditions are satisfied only in the centers of boundary elements. In all other points of the border, for the method of domain discretization, the boundary conditions may not be met. Secondly, the contour integrals in the expressions of velocity (18) and (18) and (19) (Katsikadelis 2002). Also, to increase the accuracy of the solution during numerical approximation, nonlinear functions may be used (often polynomials), describing the velocity and surface forces fora boundary element. Then, of course, the analytical calculation of integrals (37) and (38) is complex or impossible. For the calculation of integrals (37) and (38), numerical methods can also be used. However, in this case, the calculation time and demand for space in the computer's memory increase. 
Fig. 4 and 5 show the graphs of the velocity components $v_{x}, v_{y}$ in sectional views (for $x=0.5, y=0.5$ ) calculated by the BEM for 4000 elements (dotted line) and by the finite element method (FEM) (solid line). In this case, the solution obtained by the FEM is treated as similar to the exact solution.

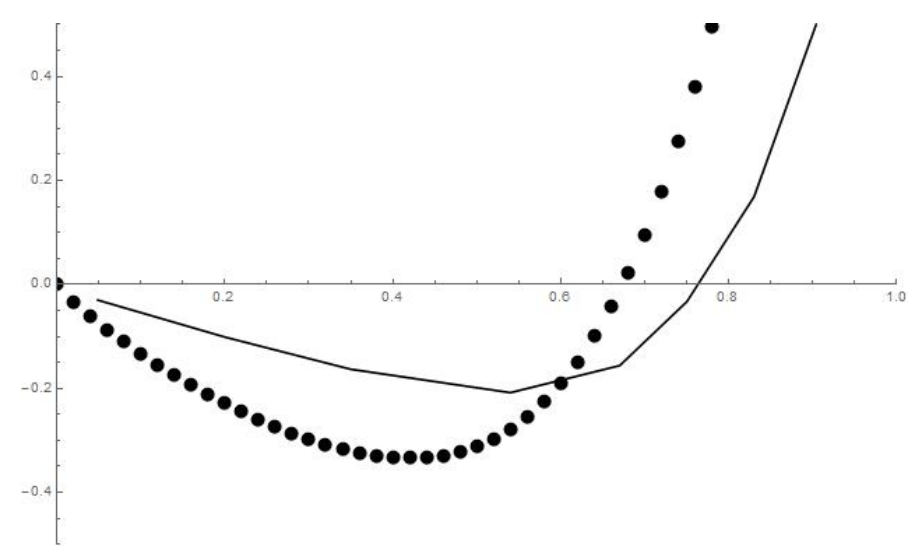

Fig. 4. Velocity component $v_{x}$ in section $x=0.5$ (FEM - solid line, BEM - dotted line)

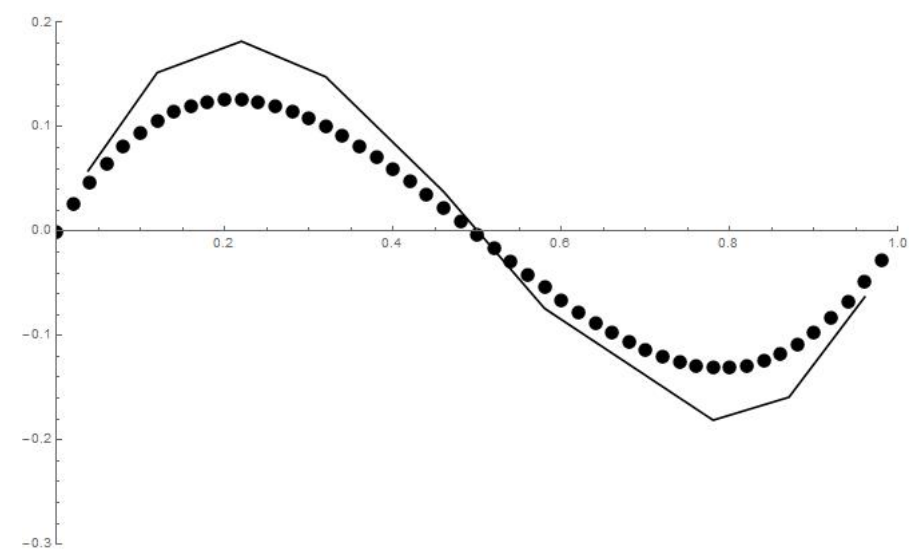

Fig. 5. Velocity component $v_{y}$ in section $x=0.5$ (FEM - solid line, BEM - dotted line)

Comparing the solutions obtained by the FEM and BEM (Fig. 4 and 5), we can see that the error of the BEM is relatively high because of the method adopted for the numerical approximation of integral equations. In contrast, Teleszewski and Sorko (2011) show the results of a solution obtained for a similar case, in which the error of the BEM does not exceed $1 \%$ for 400 boundary elements and $0.5 \%$ for 800 elements. 


\section{Summary and Conclusions}

This paper presents the application of an indirect variant of the boundary element method to solve the two-dimensional steady flow of a Stokes liquid. The numerical simulations of flow in a square cavity with one moving wall were analyzed. The analysis was based on a numerical solution obtained by the finite element method and the boundary element method.

In conclusion, it should be pointed out that the application of the boundary element method for solving the steady flow of a Stokes liquid is forward-looking. Conversion of differential equations to integral equations makes it possible to reduce the dimensions of the domain subject to discretization, which leads to an easier process of digitization and a smaller number of discrete components. The ease of using the BEM for multi-coherent areas with non-trivial geometry makes this method more forward-looking than popular methods, such as the finite element method or the finite difference method. The advantages of the BEM may also include its application to solving the problems of three-dimensional flows of a Stokes liquid. However, when applying the BEM, particular attention should be paid to the method used for the numerical approximation of basic equations. The choice of the approximation method has an impact on such things as the choice of the solution algorithm, the accuracy of the solution, the number of elements used during discretization, computation time, and the required amount of computer memory needed to perform calculations.

\section{References}

Banerjee P. K., Butterfield R. (1981) Boundary Element Methods in Engineering Science, McGraw-Hill, London, New York.

Brebbia C. A. (1982) Boundary Element Methods in Engineering, Springer-Verlag, Berlin.

Childress S. (2008) An Introduction to Theoretical Fluid Dynamics. Chapter 7 - Stokes flow, New York.

Hahn M. (2013) Investigation of the boundary integral method for Stokes flow, Master's thesis: University of Bayreuth, Bayreuth.

Katsikadelis J. T. (2002) Boundary Elements: Theory and Applications, Elsevier Science, Ron Mobed, Oxford.

Marin O. (2012) Boundary integral methods for Stokes flow: Quadrature techniques and fast Ewald methods, PhD thesis: KTH Royal Institute of Technology, Stockholm.

Pozrikidis C. (1992) Boundary Integral and Singularity Methods for Linearized Viscous Flow, Cambridge University Press, Peter Phillips, Cambridge.

Teleszewski T. J., Sorko S. A. (2011) Application of the boundary element method for the simulation of two-dimensional viscous flow, Acta Mechanica et Automatica, 5 (1) (in Polish). 